\title{
Potential function of microRNAs in thoracic aortic aneurysm and thoracic aortic dissection pathogenesis
}

\author{
TUGCE SENTURK ${ }^{1}$, ARZU ANTAL ${ }^{2}$ and TUBA GUNEL ${ }^{1}$ \\ ${ }^{1}$ Department of Molecular Biology and Genetics, Istanbul University, Istanbul 34134; \\ ${ }^{2}$ Cardiovascular Surgery Clinic, Kartal Kosuyolu Training and Research Hospital, Istanbul 34865, Turkey
}

Received February 9, 2019; Accepted September 9, 2019

DOI: $10.3892 / \mathrm{mmr} .2019 .10761$

\begin{abstract}
Thoracic aortic aneurysm (TAA) and thoracic aortic dissection (TAD) are aortic diseases known as 'silent killers'. While TAA is characterized by an enlargement of at least half of the normal aortic diameter, TAD is characterized by progressive pseudo-lumen formation, which results in the gradual separation of the aortic wall layers. In the present study, a total of 28 serum samples from nine patients with TAA, nine patients with TAD and ten healthy individuals were studied. The aim of the present study was to investigate the expression profiles of hsa-microRNA(miR)-143-3p and hsa-miR-22-3p in TAA and TAD in order to identify candidate miRNAs that are responsible for the pathogenesis of the diseases. Following the detection of target mRNAs from candidate miRNAs by bioinformatic tools, the expression profiles of target mRNAs were analyzed. A quantitative polymerase chain reaction was performed to detect Kirsten rat sarcoma viral oncogene homolog (KRAS), mitogen-activated protein kinase (MAPK) 7, MAPK14 and transgelin (TAGLN) mRNA expression profiles. The results of the comparison with control group demonstrated that the increase in the expression levels of hsa-miR-143-3p ( $\mathrm{P}=0.017)$ and hsa-miR-22 $(\mathrm{P}=0.03)$ candidate miRNAs were statistically significant in the TAA group, but not in the TAD group. The expression of KRAS and MAPK7 mRNAs decreased in the two groups compared with the control group. The level of expression of MAPK14 decreased in the TAD group, but increased in the TAA group compared with the control group. TAGLN mRNA expression level increased in the two groups. The statistically significant difference in the expression of hsa-miR-143-3p suggests that hsa-miR-143-3p may be a potential biomarker for TAA, as the expression of the target mRNAs KRAS and MAPK7 decreased
\end{abstract}

Correspondence to: Professor Tuba Gunel, Department of Molecular Biology and Genetics, Istanbul University, Sehzadebas1 Street, Istanbul 34134, Turkey

E-mail: gunel@istanbul.edu.tr

Key words: thoracic aortic aneurysm, thoracic aortic dissection, microRNAs, Kirsten rat sarcoma viral oncogene homolog gene, mitogen-activated protein kinase 7 gene, transgelin gene and the miRNA-mRNA association was negatively correlated. These miRNAs and their associated genes may serve important functions in TAA formation, the altered expression of which may be important in the pathogenesis of TAA.

\section{Introduction}

Thoracic aortic diseases, including aneurysms and dissections of the thoracic aorta, are a major cause of morbidity and mortality in developed countries (1). The incidence of aortic diseases, such as thoracic aortic aneurysm (TAA) and thoracic aortic dissection (TAD), has been estimated at 6 per 100,000 individuals/year (2). TAAs are localized dilatations of the supra-diaphragmatic aorta that result from the weakening and expansion of the arterial wall (3). TAD is characterized by the separation of the thoracic aortic wall layers by extraluminal blood that typically enters the vessel wall via an intimal tear (4). The primary cause of aortic diseases is the degeneration of the aortic media layer due to hereditary and environmental factors (5). The process of the degeneration of the media layer, consisting of elastin and smooth muscle cells (SMC) involves changes to the SMC phenotype, loss of elastin layers and the increased production of matrix metalloproteinases and proteoglycans (6).

MicroRNAs (miRNAs/miRs) are small non-coding single-stranded RNAs ( 21 nucleotides in length) that negatively regulate or repress target gene expression. miRNAs regulate gene expression in numerous physiological mechanisms, in addition to pathological conditions within the cells (7). A number of miRNAs have been identified that are associated with TAAs and TADs (8). Hsa-miR-143-3p is one of the most important miRNAs expressed by vascular SMCs (VSMC) and interacts with Kirsten rat sarcoma viral oncogene homolog (KRAS) and mitogen-activated protein kinases (MAPKs), associated with the pathogenesis of aortic diseases $(9,10)$. This miRNA regulates the MAPK pathways that induce TAA formation via non-canonical transforming growth factor $\beta$ signaling (11). The KRAS gene transcription product is the K-RAS protein, which is the part of a signaling pathway known as RAS type GTPase family/MAPK pathway (12). Another miRNA, hsa-miR-22-3p, has been associated with MAPK14, which is implicated in VSMC differentiation, proliferation, migration and fibrosis (13). In addition to these, SMCs from thoracic aneurysms are characterized by the decreased expression of SMC contractile proteins, including transgelin (TAGLN) (14). 
In the present study, hsa-miR-143-3p and hsa-miR-22-3p expression profiles were compared between patients with TAA and TAD, and healthy volunteers. The present study also investigated the potential target genes of these miRNAs. Selected KRAS, MAPK7, MAPK14 and TAGLN genes expression analyses were performed. In addition, the present study performed pathway analysis for each miRNA-mRNA pair.

\section{Materials and methods}

Study design and subjects. The present study included a total of 28 serum samples obtained from 9 patients with TAA, 9 patients with TAD and 10 healthy individuals. Patients who had been operated on for TAA $(n=9)$ and TAD $(n=9)$ between 2017 and 2018 at the Cardiovascular Surgery Clinic of Kartal Kosuyolu Heart Research Hospital (Istanbul, Turkey) were enrolled in the present study. A total of 9 patients were diagnosed with acute Stanford type A aortic dissection and received surgery on an emergency basis, while 9 patients were diagnosed with an ascending aortic aneurysm, and received surgery electively. The experimental group refers to both groups of patients. Patient data were collected from clinical records of the hospital prospectively, following the recieval of ethical approval of the study by the Ethics Committee of Istanbul University. All patients provided written informed consent to participate in the study. All patients with acute aortic dissection were diagnosed via a computed tomography (CT) scan and were further evaluated with transthoracic echocardiography prior to surgery. Patients with asending aortic aneurysms were diagnosed using routine echocardiographic evaluation and were followed-up by serial CT scans until a surgical decision was made by the treating physician. A further 10 individuals were admitted to the hospital for routine examinations and were identified as healthy without any aortic pathology, and these individuals agreed to be included in the present study as the control group. The control group also received a transthoracic echocardiograhic evaluation in order to demonstrate the absence of any aortic and valvular pathology.

Sample demographics. The demographic characteristics of patients with TAA and TAD constituting the experimental groups, and the healthy individuals constituting the control group, are presented in Table I. In all groups (experimental and healthy), individuals with Marfan syndrome, Loeys-Dietz syndrome, aneurysm osteoarthritis syndrome, arterial gangrene syndrome and Ehlers Danlos syndrome were excluded from the present study. Patients with syndromic thoracic aortic aneurysms, known or suspected connective tissue disorders, and patients with bicuspid aortic valves were not included in the present study. Patients with inflammatory, autoimmune diseases and traumatic etiology were not included in the present study. The present study did not include patients $>65$ years old in the TAA and control groups. All patients were male, with a mean age of $47 \pm 11$ years (range, 22-63 years) in the acute dissection group; mean $58 \pm 4$ years (range, $45-65$ years) in the asending aortic aneurysm group; and mean 53 \pm 1 years (range, $42-63$ years) in the control group.
Bioinformatics analysis. Bioinformatic tools were used in the present studyin order to not only determine candidate miRNAs and their subsequent sequences, but also to determine the target genes associated with these miRNAs and sequences. The present study aimed to decrease the rate of false positivity in the bioinformatic tools used by making use of features including seed matching, conservation, free energy and region accessibility to predict miRNA-mRNA interaction. To identfy putative target mRNAs, the miRGator bioinformatic tool (http://mirgator.kobic.re.kr/) was used which shows the comparative results of other bioinformatics tools such as microRNA. org (http://www.microrna.org/microrna/home.do), miRBase (http://www.mirbase.org/), PITA (https://www.mybiosoftware.com/pita-6-microrna-prediction-tool.html) and PicTar (https://pictar.mdc-berlin.de/). Also, TargetScan (http://www. targetscan.org/vert_72/), mirDB (http://www.mirdb.org/) and RNAHybrid (https://bibiserv.cebitec.uni-bielefeld.de/rnahybrid) bioinformatic tools were used to confirmation target mRNAs found by miRGator. In addition, Pathway Studio ${ }^{\circledR}$ and ENSEMBL were used for the pathway analysis of candidate miRNAs and their target mRNA (details nad URLs are presented in Table II).

Biological samples and total RNA extraction. Peripheral blood samples were collected from healthy individuals and patients. Blood samples were transferred to the Istanbul University Molecular Biology and Genetics Department from Cardiovascular Surgery Clinic at Kartal Kosuyolu Training and Research Hospital within $36 \mathrm{~h}$ at the latest using the cold chain method and stored at $4^{\circ} \mathrm{C}$ until the genetic analyses were performed.

The peripheral blood samples were centrifuged at $937 \mathrm{x} \mathrm{g}$ for $20 \mathrm{~min}$ at $4^{\circ} \mathrm{C}$ and the supernatant was transferred into microcentrifuge tubes. Serum was then aliquoted and stored at $-80^{\circ} \mathrm{C}$ for long-term storage. Total RNA, including miRNA, was extracted from the serum using an mirVana PARIS kit (Ambion; Thermo Fisher Scientific, Inc., Waltham, MA, USA) according to the manufacturer's protocol. The concentrations of the RNA were determined on the basis of the absorbance at $260 \mathrm{~nm}$, and the purity was assessed on the basis of the absorbance ratio at 260/280 $\mathrm{nm}$ using a NanoDrop spectrophotometer. The samples were preserved as total RNA at $-80^{\circ} \mathrm{C}$ until use for the reverse transcription-quantitative polymerase chain reaction (RT-qPCR).

miRNA and mRNA analyis via $R T-q P C R$. The present study used RT-qPCR in the analyis of the expression levels of the 2 miRNAs and mRNA of 4 genes. Each serum sample was analyzed for the following miRNAs and mRNAs using gene-specific TaqMan primer/probe sets (Applied Biosystems; Thermo Fisher Sceintific, Inc.): Hsa-miR-143-3p (assay ID: 002249), hsa-miR-22-3p (assay ID: 000398), KRAS (assay ID: Hs00364284_g1), MAPK7 (assay ID: Hs0061114_ g1), MAPK14 (assay ID: Hs01051152_m1) and TAGLN (assay ID: Hs01038777_g1). Synthetic cel-miR-39 and GAPDH served as the internal controls for the normalization of the miRNA and mRNA expressions analyses. Negative PCR controls were run to verify the absence of genomic DNA contamination.

For the miRNA expression analysis, selected miRNAs underwent RT to form complementary DNA (cDNA) using 
Table I. Patients and healthy controls demographics.

\begin{tabular}{lccc}
\hline & \multicolumn{3}{c}{ Study groups } \\
\cline { 2 - 3 } Demographic characteristics & TAA & TAD & Control group \\
\hline Number of patients/individuals & 9 & 9 & 10 \\
Mean age, years & 58.4 & 47.1 & 53.1 \\
Mean height, cm & 169 & 85 & 173 \\
Mean weight, kg & 89.8 & $7(77.7)$ & 80 \\
Hypertension, $\mathrm{n}(\%)$ & $9(100)$ & $1(11.1)$ \\
Diabetes, $\mathrm{n}(\%)$ & - & $1(11.1)$ & $6(66.6)$ \\
COPD, $\mathrm{n}(\%)$ & - & - & - \\
Smoking, $\mathrm{n}(\%)$ & $2(22.2)$ & $1(11.1)$ & - \\
CVD, $\mathrm{n}(\%)$ & - & $1(11.1)$ & - \\
Obesity, $\mathrm{n}(\%)$ & & - \\
\hline
\end{tabular}

COPD, choronic obstructive pulmonary disease; CVD, cerebrovascular disease; TAA, thoracic aortic aneurysm; TAD, thoracic aortic dissection.

Table II. Table of bioinformatic tools utilized in the present study.

\begin{tabular}{|c|c|c|c|}
\hline Tool name & Purpose & Features & Website (URL) \\
\hline TargetScan & $\begin{array}{l}\text { Target gene prediction by } \\
\text { seed region sequence }\end{array}$ & Seed match, Conservation & www.targetscan.org \\
\hline $\operatorname{mirDB}$ & $\begin{array}{l}\text { Target gene prediction by } \\
\text { 3'UTR and CDS }\end{array}$ & $\begin{array}{l}\text { Seed match, conservation, } \\
\text { free energy, site accessibility }\end{array}$ & www.mirdb.org \\
\hline RNAHybrid & $\begin{array}{l}\text { Target gene prediction by } \\
\text { secondary structure matching }\end{array}$ & Seed Match, Free Energy & $\begin{array}{l}\text { www.bibiserv2.cebitec. } \\
\text { unibielefeld.de/rnahybrid }\end{array}$ \\
\hline miRGator & $\begin{array}{l}\text { miRNA sequence analysis, target } \\
\text { gene prediction and pathway } \\
\text { analysis }\end{array}$ & $\begin{array}{l}\text { Comparison of multiple } \\
\text { bioinformatics tools }\end{array}$ & http://mirgator.kobic.re.kr/ \\
\hline NCBI & $\begin{array}{l}\text { Detection of miRNA and target } \\
\text { genes sequences }\end{array}$ & $\begin{array}{l}\text { Access to genomic } \\
\text { information }\end{array}$ & https://www.ncbi.nlm.nih.gov/ \\
\hline ENSEMBL & Target gene functions & $\begin{array}{l}\text { Explanation for target genes } \\
\text { sequence and structure }\end{array}$ & https://www.ensembl.org/index.html \\
\hline $\begin{array}{l}\text { PATHWAY } \\
\text { STUDIO }\end{array}$ & Pathway analysis & $\begin{array}{l}\text { Allowance for analysis } \\
\text { and visualization of } \\
\text { disease mechanisms, gene } \\
\text { expression and proteomics } \\
\text { and metabolomics data }\end{array}$ & https://www.pathwaystudio.com/ \\
\hline
\end{tabular}

a TaqMan MicroRNA Reverse Transcription kit (Ambion; Thermo Fisher Scientific, Inc.) according to the manufacturer's protocol. The RT product was amplified using gene-specific TaqMan primer/probe sets and the TaqMan Universal PCR Master Mix II (Ambion; Thermo Fisher Scientific, Inc.) with uracil-N glycosylase by the following a 2-step procedure: Initial denaturation for $10 \mathrm{~min}$ at $95^{\circ} \mathrm{C}$, followed by 40 cycles of $15 \mathrm{sec}$ at $95^{\circ} \mathrm{C}$, and $60 \mathrm{sec}$ at $60^{\circ} \mathrm{C}$.

First, for the mRNA expression analysis, cDNA was synthesized from total RNA using a High Capacity cDNA Reverse Transcription kit and random primers (Agilent SureCycler 8800; Agilent Technologies, Inc., Santa Clara, CA, USA). cDNA was used to analyze specific gene expression with specific Taqman Gene Expression Assays on a CFX96 Touch $^{\mathrm{TM}}$ Real-Time PCR detection system. The thermal cycling protocol was performed as follows: 2 min at $95^{\circ} \mathrm{C}$, $2 \mathrm{~min}$ at $95^{\circ} \mathrm{C}$, followed by 40 cycles of $95^{\circ} \mathrm{C}$ for $15 \mathrm{sec}$, and $60^{\circ} \mathrm{C}$ for $1 \mathrm{~min}$.

Data analysis. All statistical analyses were performed using GraphPad Prism (version 7.04; GraphPad Software, Inc., La Jolla, CA, USA). The Cq values of the samples were automatically determined on a CFX96 Touch $^{\mathrm{TM}}$ Real-Time PCR. The expression level of each miRNA and mRNA was calculated using the $2^{-\triangle \Delta C q}$ method (15). Data were analyzed for outliers. D'Agostino \& Pearson's normality test was performed in order 
A

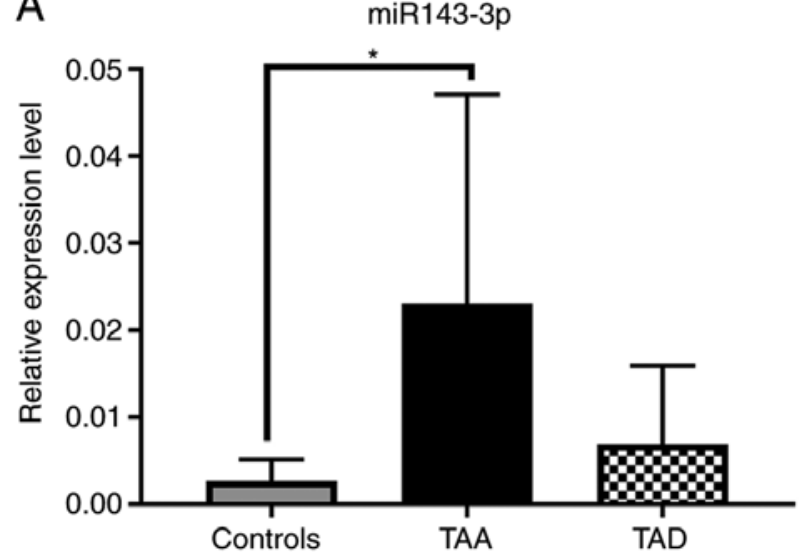

B

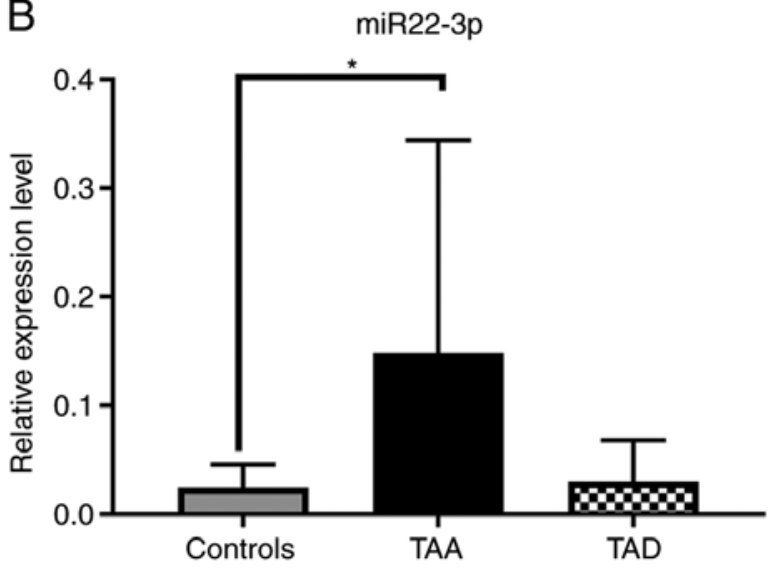

Figure 1. (A) Expression levels of hsa-miR-143-3p, and (B) hsa-miR-22-3p. * $\mathrm{P}<0.05$. Values are presented as the mean \pm standard error of the mean. ' $\mathrm{n}$ ' values 10 (controls), 9 (TAA), 9 (TAD). TAA, thoracic aortic aneurysm; TAD, thoracic aortic dissection; miR, microRNA.

to determine whether the data were parametric or non-parametric. All data were nonparametric and comparisons between different groups were performed using Kruskal-Wallis tests. Dunn's Multiple Comparison test was performed to determine differences between specific groups. Results are presented as the mean \pm standard error of the mean. Receiver operating characteristic curves were generated, and the area under the curve (AUC), sensitivity and specificity were calculated to evaluate the diagnostic values of candidate miRNAs and mRNA of targeted genes. Correlation analysis was performed using a Pearson correlation test. $\mathrm{P}<0.05$ was considered to indicate a statistically significant difference.

\section{Results}

miRNA expression analysis. The expression level of hsa-miR-143-3p increased in the TAA and TAD groups compared with the control group. While this increase was statistically significant in the TAA group when compared with the control group $(\mathrm{P}<0.05)$, there was no significant difference observed in the TAD group compared with the control group. The expression level of hsa-miR-22-3p increased in the two experimental groups when compared with the control group. While this increase was statistically significant in TAA group when compared with the control group $(\mathrm{P}<0.05)$, no significant difference was observed in the TAD group when compared with the control group (Fig. 1).

Hsa-miR-143-p and hsa-miR-22-3p exhibited a diagnostic value in differentiating patients with TAA from healthy volunteers, with AUC values of 0.92 and 0.80 , respectively. Hsa-miR-143-p with AUC values of 0.60 exhibited diagnostic value when differentiating patients with TAD from healthy volunteers. The present study also demonstrated that the AUC values of hsa-miR-22-2p were not useful as a diagnostic tool when differentiating patients with TAD from healthy volunteers. The receiver operating characteristic (ROC) curves analysis of this miRNA revealed that the AUC was 0.50 (Fig. 2).

Determination of target genes. Tarbase, MirTarbase, TargetScan, MiRNAorg, PITA, PicTar and MirDB were selected as target prediction tools. These tools demonstrated that hsa-miR-143-3p and hsa-miR-22 may target certain associated genes, including KRAS, MAPK7, MAPK14 and TAGLN. As a result of comparing the different computer programs and databases, MAPK7, MAPK14 and TAGLN were predicted by at least five of the seven algorithms, and KRAS was predicted by six of the algorithms (data not shown).

mRNA expression analysis. The expression levels of KRAS and MAPK7 target mRNAs were decreased in the TAA and TAD groups, but this was not statistically significant $(\mathrm{P}>0.05)$. The expression of MAPK14 mRNA was increased in the TAA group, but decreased in the TAD groups, however, this increase and decrease was not statistically significant. The level of expression of TAGLN was increased in each of the patient groups, but was not significant. However, the expression levels of TAGLN were 40-fold higher in the TAD group compared with the control group (Fig. 3).

The ROC curves analysis of these mRNAs demonstrated (Fig. 4) that MAPK14 exhibited diagnostic value in differentiating each of the patient groups from the healthy volunteers, with AUC values of 0.7 and 0.8 in the TAA and TAD groups, respectively. TAGLN with AUC values of 0.8 exhibited diagnostic value when differentiating patients with TAD from healthy volunteers.

Correlation analysis between miRNA and target genes. The present study investigated whether miR levels were correlated with their respective mRNA targets. The positive and negative correlations are summarized in Fig. 5. A negative correlation between hsa miR-22-3p and TAGLN $\left(\mathrm{r}^{2}=-0.46 ; \mathrm{P}=0.18\right)$ and positive correlation between hsa-miR-22-3p and MAPK14 $\left(\mathrm{r}^{2}=0.09 ; \mathrm{P}=0.087\right)$ was observed. A negative correlation was observed between hsa-miR-143-3p and KRAS $\left(r^{2}=-0.36\right.$; $\mathrm{P}=0.37)$ and MAPK $7\left(\mathrm{r}^{2}=-0.3 ; \mathrm{P}=0.57\right)$. All correlation analysis results were not statistically significant $(\mathrm{P}>0.05)$.

\section{Discussion}

TAA and TAD are the most common aortic diseases to be diagnosed following an extended period of subclinical development or presentation of an acute complication (16). TAA, 

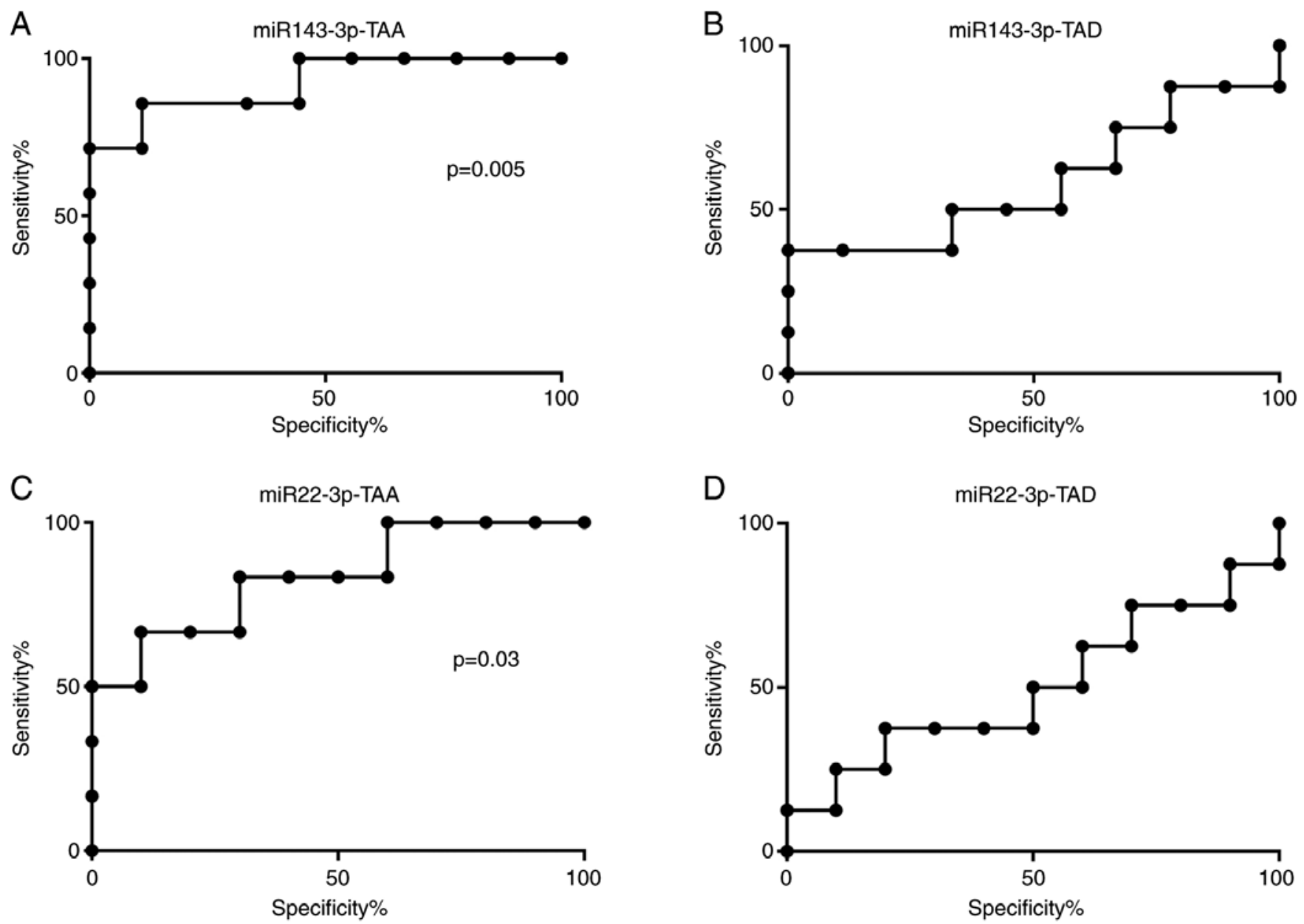

Figure 2. ROC curves of serum miRNAs levels. (A) ROC analysis of hsa-miR-143-3p in the TAA group, AUC=0.92. (B) ROC analysis of hsa-miR-143-3p in the TAD group. (C) ROC analysis of hsa-miR-22-3p in the TAA group, AUC $=0.8$ (D) ROC analysis of hsa-miR-22-3p in the TAD group. $\mathrm{P}<0.05$ was considered to indicate a statistically significant difference. MiRNA/miR, microRNA; TAA, thoracic aortic aneurysm; TAD, thoracic aortic dissection; AUC, area under the curve; ROC, receiver operating characteristic.

which usually progresses in an asymptomatic manner, is not noticeable and can result in dissection, rupture or mortality if left untreated (1). According to Kent (17), the majority of patients with aortic rupture are unlikely to survive, so it is necessary to identify the aneurysms prior to this stage being reached. The limitations in the diagnosis and treatment options currently available increase the necessity of investigating clinically important biomarkers for the early diagnosis of the disease with a noninvasive approach (18). TAD is a rare but life-threatening condition with a lethality rate of $1-2 \%$ per hour following the onset of symptoms in untreated patients. Therefore, prompt and proper diagnoses are vital in order to increase the patient's chance of survival and to prevent grievous complications (19).

miRNAs are small non-coding RNA molecules that are involved in the regulation of target mRNA molecules and are detectable in tissue, blood or urine samples. One previous characterization study confirmed that circulating miRNAs are stable in plasma and serum (20). The miRNA expression profile was evaluated in the clinical patient samples due to their function in the pathogenesis of TAD and TAA.

The aim of the present study was, first, to determine the differences in the expression of hsa-miR-143-3p and hsa-miR-22-3p in serum levels of TAA $(n=9)$ and TAD $(n=9)$ groups compared with healthy controls $(n=10)$. Secondly, the aim was to determine those mRNAs targeted by candidate miRNAs with network-based bioinformatics programs and algorithms. Thirdly, to reveal the changes in the expression levels of the mRNAs, detected by qPCR. The validation of hsa-miR-143-3p and hsa-miR-22-3p, which were selected as candidate miRNA following microarray studies in TAA and TAD groups $(4,21,22)$, were performed in the present study.

Hsa-miR-143-3p is a highly expressed miRNA in VSMC. The gene encoding hsa-miR-143-3p, which is localized in the $5 \mathrm{q} 32$ region of the human genome, is highly conserved among species (23). It has been demonstrated that they serve important functions in the phenotypic change of VSMCs and in regulating the pathogenesis of vascular diseases (24). Its functions include targeting numerous transcription factors, including Kruppel-like factor-41, myocardin and ETS Transcription Factor, and induces the differentiation of VSMCs and suppresses their proliferation (21). According to Climent et al (25), hsa-miR-143-3p regulates the vessel stabilization properties of endothelial cells by functioning as a communication molecule between SMCs and endothelial cells. Hsa-miR-143-3p inhibits cell proliferation by targeting KRAS and extracellular signal regulated kinase (ERK)5 directly (Fig. 6) (26). 

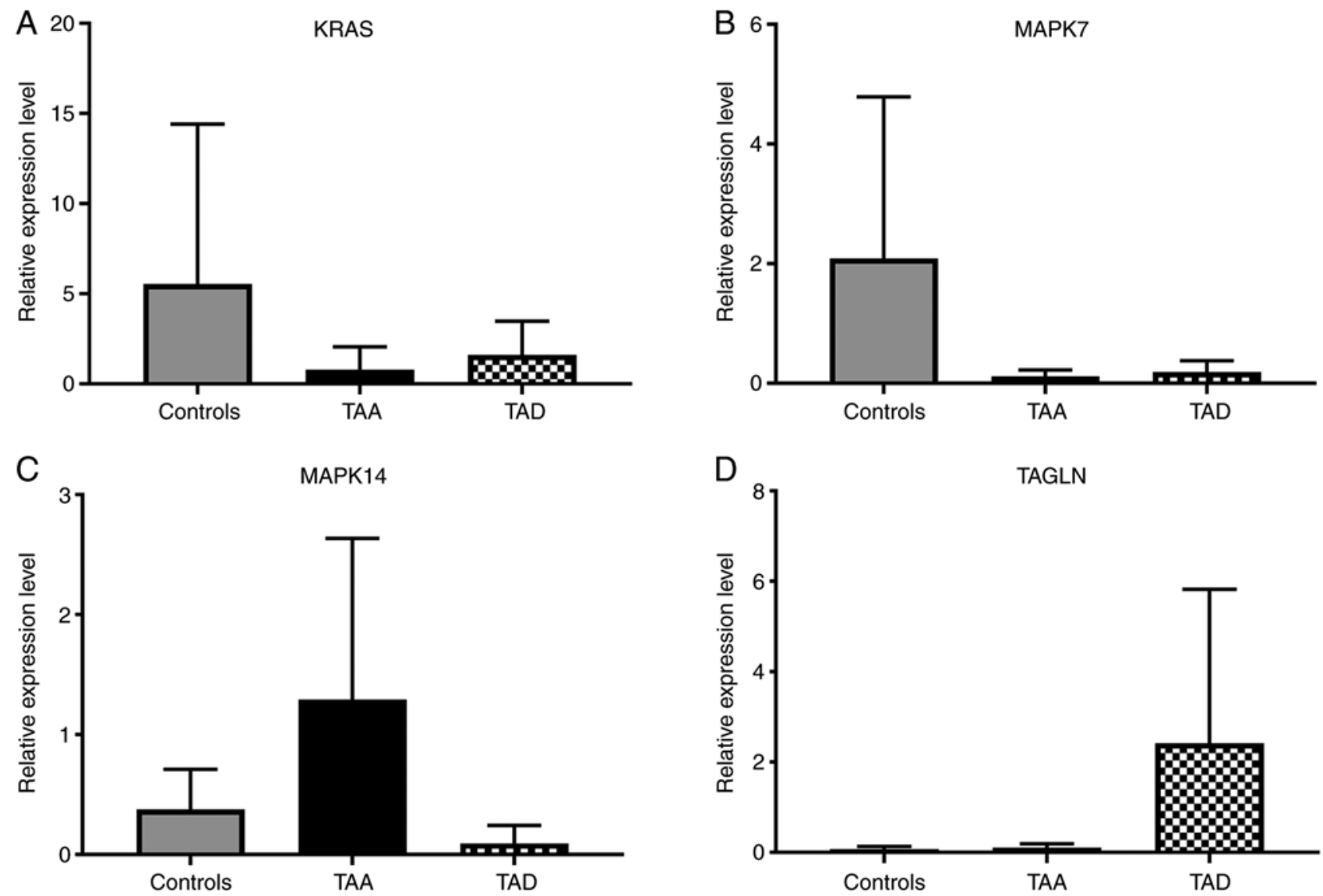

Figure 3. Expression levels of genes targeted by hsa-miR-143-3p and hsa-miR-22 in TAA, TAD and control cells. (A) Expression levels of KRAS, (B) MAPK7, (C) MAPK14 and (D) TAGLN. Values are presented as the mean \pm standard error of the mean. 'n' values 10 (controls), 9 (TAA), 9 (TAD). TAA, thoracic aortic aneurysm; TAD, thoracic aortic dissection; KRAS, Kirsten rat sarcoma viral oncogene homolog; MAPK, mitogen-activated protein kinase; TAGLN, transgelin.

In the present study, the expression levels of KRAS mRNA, which is the target gene of hsa-miR-143-3p, decreased by 7-fold in the TAA group and 3.5-fold in the TAD group when compared with the control group. Wang et al (27) demonstrated that KRAS is a target of miR-143. The KRAS/ERK pathway is one of the most important pathways associated with the proliferation of VSMCs. The accumulation of VSMCs migrating from media to the intima in the aortic wall and resulting in proliferation results in vascular remodelling (28). VSMC proliferation is necessary for diseases characterized by ballooning in the aortic structure (29). Hsa-miR-143-3p regulates VSMC proliferation by inhibiting KRAS. The increased expression level of hsa-miR-143-3p results in a decrease in KRAS expression and VSMC proliferation. These results may indicate that hsa-miR-143-3p is a regulator of VSMC proliferation with KRAS (28).

It was observed, in the present study, that the expression level of MAPK7, which was determined and validated as a target of hsa-mir-143-3p via bioinformatics tools, decreased 17-fold in the TAA group and 11-fold in the TAD group when compared with the control group. Osaki et al (10) demonstrated that MAPK7 is the target of hsa-miR-143-3p, while Roberts et al (30) revealed that endothelial cells increased the survival rate of VEGF-mediated MAPK7 (ERK5). Decreased MAPK7 expression increased the apoptosis rate of VSMC and endothelial cells, causing functional changes in the aortic wall structure. Modifications of endothelial cells may induce degradation of aortic wall structure by affecting VSMCs (30).

The level of expression of another candidate miRNA, hsa-miR-22-3p, was observed to be significantly higher compared with the control group, with a 6-fold increase in the TAA group, but no statistically significant difference in the TAD group, with a 1.5 -fold increase, compared with the control group. Hsa-miR-22-3p is localized on the long arm of chromosome 17 in region p13.3 of the human genome according to the National Center for Biotechnology Information. According to Liao et al (4), the decrease in the expression levels of hsa-miR-22-3p were statistically significant in patients with TAD when investigated via microarray and $\mathrm{qPCR}$ processes. Patuzzo et al (21) demonstrated that the expression level of hsa-miR-22-3p decreased by 1.5 -fold, but this decrease was not statistically significant. Hsa-miR-22-3p has been indicated to serve a function as a regulator during stem cell VSMC differentiation, but little is currently known about target genes and their associated pathways in mature VSMC phenotype modification or its clinical effects (31); it has also been reported to regulate apoptosis in cardiomyocytes by targeting MAPK14 (Fig. 7) (31).

As a result of the relative comparison, MAPK14 expression was increased 3-fold in the TAA group, and decreased 4-fold in the TAD group. The ROC analysis results for the MAPK14 gene revealed AUC values of 0.7 and 0.8 in the TAA and TAD groups, respectively. 

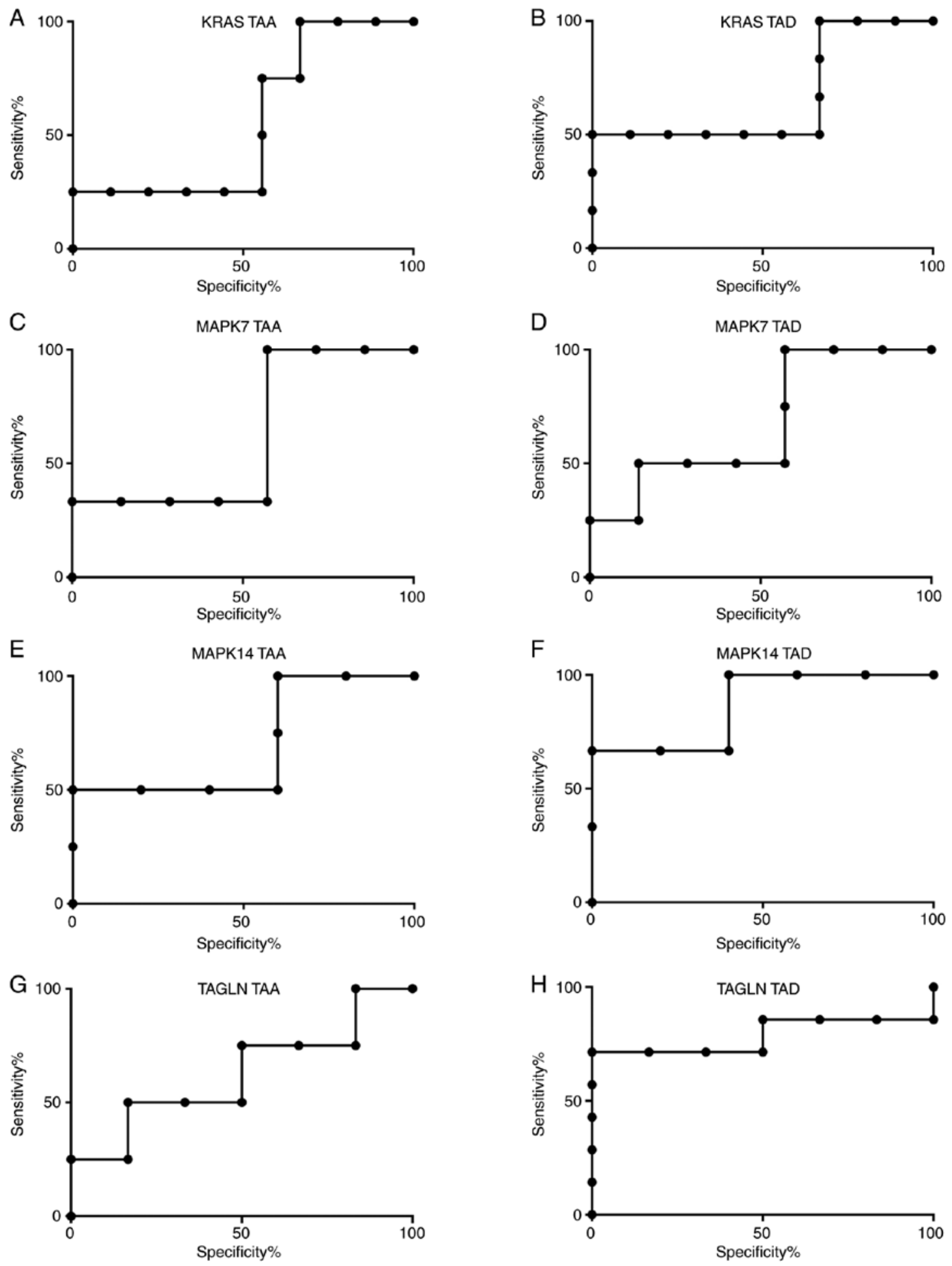

Figure 4. ROC analysis of the target genes. ROC analysis of (A) KRAS in the TAA group, (B) KRAS in the TAD group, (C) MAPK7 in the TAA group, (D) MAPK7 in the TAD group, (E) MAPK14 in the TAA group, (F) MAPK14 in the TAD group, (G) TAGLN in the TAA group and (H) TAGLN in the TAD group. TAA, thoracic aortic aneurysm; TAD, thoracic aortic dissection; ROC, receiver operating characteristic; KRAS, Kirsten rat sarcoma viral oncogene homolog; MAPK, mitogen-activated protein kinase; TAGLN, transgelin.

A statistically significant increase in hsa-miR-22-3p may result in the decreased expression of the MAPK14 gene in the TAA group. As a result of the ROC analysis, high AUC values obtained in the TAD and TAA groups indicated that hsa-miR-22-3p and target mRNA MAPK14 may be an important biomarkers for clinical tests. Decreases in MAPK14 mRNA expression in patients with TAA may result in aneurysms due to the decreased VSMC contractile phenotype. However, another study performed by the same study group revealed that if p38MAPK suppressed the dominant isoform MAPK14, the markers including VSMCs and TAGLN in the contractile phenotype increased (32). Increased levels of MAPK14 mRNA expression in the TAD group may result in a decreased VSMC contractile phenotype. Considering that the 

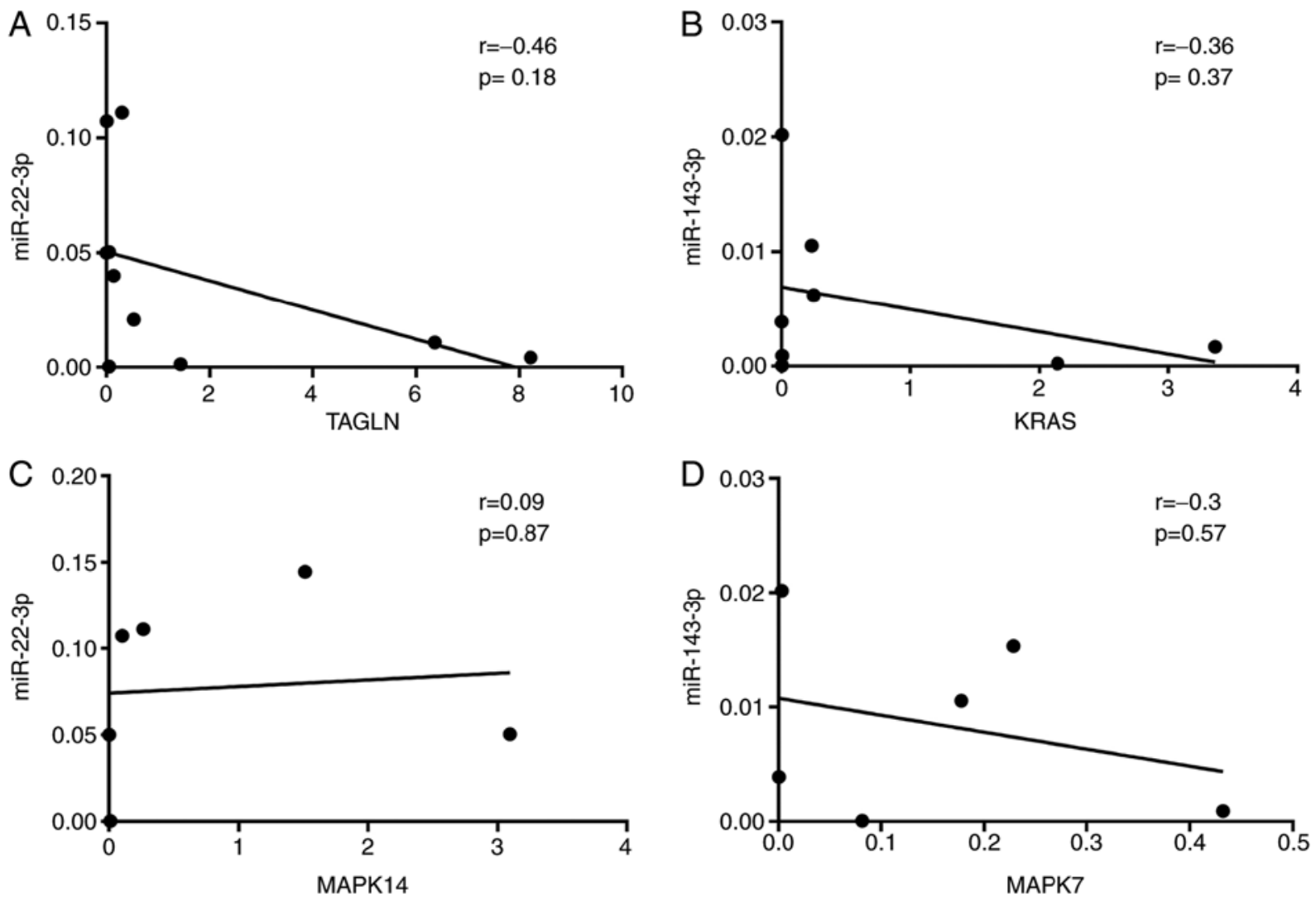

Figure 5. Correlation between miR-22-3p and miR-13-3p and their target gene mRNA. (A) miR-143 vs. KRAS, (B) miR-143 vs. MAPK7, (C) miR-22 vs. MAPK14 and (D) miR-22 vs. TAGLN. miR, microRNA; KRAS, Kirsten rat sarcoma viral oncogene homolog; MAPK, mitogen-activated protein kinase; TAGLN, transgelin.

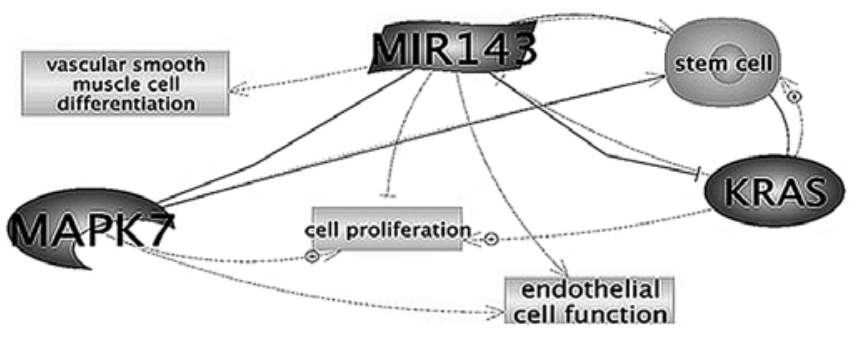

Figure 6. Pathway analysis of hsa-miR-143-3p and target mRNAs KRAS and MAPK7. miR, microRNA; KRAS, Kirsten rat sarcoma viral oncogene homolog; MAPK, mitogen-activated protein kinase.

TAD group did not include patients with aneurysms, MAPK14 may be an important biomarker in distinguishing between patients with TAA and those with TAD due to the difference in expression levels of this gene between these patient groups.

TAGLN gene expression level increased 1.5- and 40.0-fold in the TAA and TAD groups, respectively, in the present study. As a result of the ROC analysis of the TAGLN target gene, the AUC values were calculated as 0.6 and 0.8 in the TAA and TAD groups, respectively.

TAGLN is an early distinguishing marker of smooth muscle progenitor cell differentiation, and low levels of the TAGLN protein have been detected in non-differentiated multipotent access progenitor cells. Increased expression of TAGLN in mesenchymal stem cells is considered as evidence that mesenchymal stem cells differ in an SMC phenotype (33).

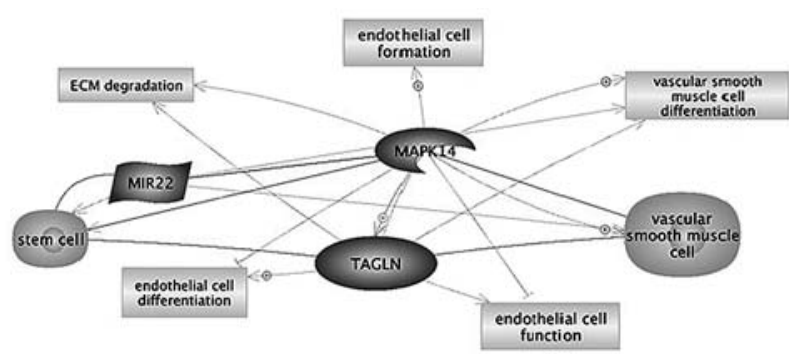

Figure 7. Pathway analysis of hsa-mir-22-3p and target mRNAs MAPK14 and TAGLN. miR, microRNA; MAPK, mitogen-activated protein kinase; TAGLN, transgelin.

It has been demonstrated that the overexpression of miR-22 in stem cells promotes SMC differentiation in vivo (33). All of these studies indicate that the activation of the injured tissue, and increase in the amount of TAGLN mRNA through inducing the differentiation of VSMCs in stem cells, causes the increased expression of hsa-miR-22-3p.

In the scope of the present study, investigating fresh serum samples obtained from patient groups is necessary in order to reflect the real situation in terms of identifying biomarkers that represent the pathophysiology of the diseases in question. The results from the present study revealed that the increase in expression levels of hsa-miR-143-3p and hsa-miR-22 candidate miRNAs were statistically significantly higher in the TAA group compared with the control group. Therefore, it was hypothesized that extracellular miRNA originating 
from SMC, endothelial cells or fibroblasts may result in endothelial cell dysfunction and/or aberrant vascular remodeling, which may initiate the intimal or media tear in aortic diseases including TAA. The expression of KRAS and MAPK7 mRNAs decreased by 7 - and 17-fold in the TAA group, respectively. Therefore, it may be suggested that, at the molecular level, hsa-miR-143-3p regulates VSMC proliferation and endothelial cell survival rate by targeting KRAS and MAPK7. Notably, the expression level of MAPK14 decreased in the TAD group while it increased in the TAA group. The results of the MAPK14 ROC analysis demonstrated that MAPK14 may be an important biomarker in the differentiation between TAA and TAD groups.

In conclusion, the results obtained in the present study may form a basis for further research into physiological studies to reflect the associations between hsa-miR-143-3p and hsa-miR-22-3p in TAA as biomarkers, and to target mRNA-miRNA associations. The main limitation of the present study was the small sample size, which was partly as a result of the scarcity of the diseases in question, and partly due to limited funding. Future studies should strive to include a large number of TAA and TAD samples to confirm these results. Another limitation is that the mean age is not similar in the TAA and TAD groups. One young patient (aged 22) in the TAD group is the reason for this difference, but conventional cardiovascular risk factors develop with advancing age and may attenuate the impact of polymorphism (34). Therefore, the present study did not include patients $>65$ years old in the TAA and control groups. For patients with TAD, the only exclusion criteria was presence of connective tissue disorders and syndromic diseases. Another minor limitation of the present study may be that the direct association between miRNA and the target genes was not demonstrated in an in vitro experiment. However, the aim was to validate the candidate miRNAs and their target genes. An in vitro study examining the direct association between miRNA and its target genes may be conducted in future studies. Additionally, although the result of the correlation analysis were not statistically significant, in light of this evidence there may still be an important association between the candidate miRNAs (hsa-miR-143-3p and hsa-miR-22-2p) and their target mRNAs (KRAS, MAPK7, MAPK14 and TAGLN). In addition, other experimental techniques including protein expression analysis by western blotting and in vivo/in vitro experiments on miRNA mimics may be used to validate the results of the present study obtained from gene expression analysis by RT-qPCR. Further research will strengthen these results and further determine the diagnostic and prognostic value of miRNAs as biomarkers in aortic diseases, and should investigate their function as a therapeutic target.

\section{Acknowledgements}

Not applicable.

\section{Funding}

The present study was supported by the Istanbul University Scientific Research Projects Department (grant no. 27359).

\section{Availability of data and materials}

The datasets generated and/or analyzed during the present study are not publicly available due to the datasets containing clinical data, and the authors therefore have an ethical and legal responsibility to respect the participants' rights to privacy and to protect their identity, but are available from the corresponding author upon reasonable request.

\section{Authors' contributions}

TS, TG and AA conceived and designed the study. TS and TG performed the experiments. AA was involved in the clinical study and preparation of the manuscript. TG and TS reviewed the manuscript.

\section{Ethics approval and consent to participate}

The present study was approved by the Ethics Committee of Istanbul University (Istanbul, Turkey; project no. 329785), and all patients provided written informed consent.

\section{Patient consent for publication}

Not applicable.

\section{Competing interests}

The authors declare that they have no competing interests.

\section{References}

1. Milewicz DM, Prakash SK and Ramirez F: Therapeutics targeting drivers of thoracic aortic aneurysms and acute aortic dissections: Insights from predisposing genes and mouse models. Annu Rev Med 68: 51-67, 2017.

2. Howard DP, Banerjee A, Fairhead JF, Perkins J, Silver LE and Rothwell PM; Oxford Vascular Study: Population-based study of incidence and outcome of acute aortic dissection and premorbid risk factor control: 10-year results from the Oxford Vascular Study. Circulation 127: 2031-2037, 2013.

3. Martufi G, Gasser TC, Appoo JJ and Di Martino ES: Mechano-biology in the thoracic aortic aneurysm: A review and case study. Biomech Model Mechanobiol 13: 917-928, 2014.

4. Liao M, Zou S, Weng J, Hou L, Yang L, Zhao Z, Bao J and Jing Z: A microRNA profile comparison between thoracic aortic dissection and normal thoracic aorta indicates the potential role of microRNAs in contributing to thoracic aortic dissection pathogenesis. J Vasc Surg 53: 1341-1349. e3, 2011.

5. Ikonomidis JS, Ivey CR, Wheeler JB, Akerman AW, Rice A, Patel RK, Stroud RE, Shah AA, Hughes CG, Ferrari G, et al: Plasma biomarkers for distinguishing etiologic subtypes of thoracic aortic aneurysm disease. J Thorac Cardiovasc Surg 145: 1326-1333, 2013.

6. Liu R, Lo L, Lay AJ, Zhao Y, Ting KK, Robertson EN, Sherrah AG, Jarrah S, Li H, Zhou Z, et al: ARHGAP18 protects against thoracic aortic aneurysm formation by mitigating the synthetic and proinflammatory smooth muscle cell phenotype. Circ Res 121: 512-524, 2017.

7. Boon RA, Seeger T, Heydt S, Fischer A, Hergenreider E, Horrevoets AJ, Vinciguerra M, Rosenthal N, Sciacca S, Pilato M, et al: MicroRNA-29 in aortic dilation: Implications for aneurysm formation. Circ Res 109: 1115-1119, 2011.

8. Moushi A, Michailidou K, Soteriou M, Cariolou M and Bashiardes E: MicroRNAs as possible biomarkers for screening of aortic aneurysms: A systematic review and validation study. Biomarkers 23: 253-264, 2018. 
9. Wang Y, Wu B, Dong L, Wang C, Wang X and Shu X: Circulating matrix metalloproteinase patterns in association with aortic dilatation in bicuspid aortic valve patients with isolated severe aortic stenosis. Heart Vessels 31: 189-197, 2016.

10. Osaki M, Takeshita F, Sugimoto Y, Kosaka N, Yamamoto Y, Yoshioka Y, Kobayashi E, Yamada T, Kawai A, Inoue T, et al: MicroRNA-143 regulates human osteosarcoma metastasis by regulating matrix metalloprotease-13 expression. Mol Ther 19 : $1123-1130,2011$.

11. Kim HW and Stansfield BK: Genetic and epigenetic regulation of aortic aneurysms. Biomed Res Int 2017: 7268521, 2017.

12. Jancík S, Drábek J, Radzioch D and Hajdúch M: Clinical relevance of KRAS in human cancers. J Biomed Biotechnol 2010: 150960, 2010.

13. Li Y and Maegdefessel L: Non-coding RNA contribution to thoracic and abdominal aortic aneurysm disease development and progression. Front Physiol 8: 429, 2017.

14. Ignatieva E, Kostina D, Irtyuga O, Uspensky V, Golovkin A Gavriliuk N, Moiseeva O, Kostareva A and Malashicheva A: Mechanisms of smooth muscle cell differentiation are distinctly altered in thoracic aortic aneurysms associated with bicuspid or tricuspid aortic valves. Front Physiol 8: 536, 2017.

15. Livak KJ and Schmittgen TD: Analysis of relative gene expression data using real-time quantitative PCR and the 2(-Delta Delta C(T)) method. Methods 25: 402-408, 2001.

16. Erbel R, Aboyans V, Boileau C, Bossone E, Bartolomeo RD, Eggebrecht H, Evangelista A, Falk V, Frank H, Gaemperli O, et al 2014 ESC Guidelines on the diagnosis and treatment of aortic diseases: Document covering acute and chronic aortic diseases of the thoracic and abdominal aorta of the adult. The Task Force for the Diagnosis and Treatment of Aortic Diseases of the European Society of Cardiology (ESC). Eur Heart J 35: 2873-2926, 2014.

17. Kent KC: Clinical practice. Abdominal aortic aneurysms. N Engl J Med 371: 2101-2108, 2014.

18. BurilloE,LindholtJS,Molina-SánchezP,JorgeI,Martinez-PinnaR, Blanco-Colio LM, Tarin C, Torres-Fonseca MM, Esteban M, Laustsen J, et al: ApoA-I/HDL-C levels are inversely associated with abdominal aortic aneurysm progression. Thromb Haemost 113: 1335-1346, 2015.

19. Gawinecka J, Schönrath F and von Eckardstein A: Acute aortic dissection: Pathogenesis, risk factors and diagnosis. Swiss Med Wkly 147: w14489, 2017.

20. Turchinovich A, Weiz L, Langheinz A and Burwinkel B: Characterization of extracellular circulating microRNA. Nucleic Acids Res 39: 7223-7233, 2011.

21. Patuzzo C, Pasquali A, Malerba G, Trabetti E, Pignatti P, Tessari M and Faggian G: Preliminary microRNA analysis of non syndromic thoracic aortic aneurysms. Balkan J Med Genet 15 (Suppl): S51-S55, 2012.
22. Licholai S, Blaż M, Kapelak B and Sanak M: Unbiased profile of MicroRNA expression in ascending aortic aneurysm tissue appoints molecular pathways contributing to the pathology. Ann Thorac Surg 102: 1245-1252, 2016.

23. Rangrez AY, Massy ZA, Metzinger-Le Meuth V and Metzinger L: miR-143 and miR-145: Molecular keys to switch the phenotype of vascular smooth muscle cells. Circ Cardiovasc Genet 4: 197-205, 2011.

24. Cordes KR, Sheehy NT, White MP, Berry EC, Morton SU, Muth AN, Lee TH, Miano JM, Ivey KN and Srivastava D: miR-145 and miR-143 regulate smooth muscle cell fate and plasticity. Nature 460: 705-710, 2009.

25. Climent M, Quintavalle M, Miragoli M, Chen J, Condorelli G and Elia L: TGF $\beta$ triggers miR-143/145 transfer from smooth muscle cells to endothelial cells, thereby modulating vessel stabilization. Circ Res 116: 1753-1764, 2015.

26. Song $\mathrm{B}$ and $\mathrm{Ju} \mathrm{J}$ : Impact of miRNAs in gastrointestinal cancer diagnosis and prognosis. Expert Rev Mol Med 12: e33, 2010.

27. Wang J, Lu L and Huang K: miR-143 inhibits osteosarcoma cell proliferation by downregulating K-ras expression. Int J Clin Exp Pathol 9: 3436-3441, 2016.

28. Yu ML, Wang JF, Wang GK, You XH, Zhao XX, Jing Q and Qin YW: Vascular smooth muscle cell proliferation is influenced by let-7d microRNA and its interaction with KRAS. Circ J 75: 703-709, 2011.

29. Inoue $\mathrm{T}$ and Node $\mathrm{K}$ : Molecular basis of restenosis and novel issues of drug-eluting stents. Circ J 73: 615-621, 2009.

30. Roberts OL, Holmes K, Müller J, Cross DA and Cross MJ: ERK5 is required for VEGF-mediated survival and tubular morphogenesis of primary human microvascular endothelial cells. J Cell Sci 123: 3189-3200, 2010.

31. Yang F, Chen Q, He S, Yang M, Maguire EM, An W, Afzal TA, Luong LA, Zhang L and Xiao Q: miR-22 is a novel mediator of vascular smooth muscle cell phenotypic modulation and neointima formation. Circulation 137: 1824-1841, 2018.

32. Long X, Cowan SL and Miano JM: Mitogen-activated protein kinase 14 is a novel negative regulatory switch for the vascular smooth muscle cell contractile gene program. Arterioscler Thromb Vasc Biol 33: 378-386, 2013.

33. Zhao H, Wen G, Huang Y, Yu X, Chen Q, Afzal TA, Luong le A, Zhu J, Ye S, Zhang L and Xiao Q: MicroRNA-22 regulates smooth muscle cell differentiation from stem cells by targeting methyl CpG-binding protein 2 . Arterioscler Thromb Vasc Biol 35: 918-929, 2015 .

34. Dhingra R and Vasan RS: Age as a risk factor. Med Clin North Am 96: 87-91, 2012. 